\title{
Design and Implementation of Drone in Healthcare Applications
}

\author{
Pratik Khandagale,*, Dharmaraj Eranjikal, ${ }^{* *}$, Siddhi Parab ${ }^{\text {,*** }}$, and Bhawana Garg,*** \\ ${ }^{1}$ Ramrao Adik Institute Of Technology, Nerul, Navi Mumbai
}

\begin{abstract}
A drone, in simple words, is a type of flying robot that can be remotely controlled by a human operator. It is in technology terms also known as Unmanned Aerial Vehicle (UAV). With many new advancements in drone technology, it is expected that drones may expand further to include diagnostic capabilities, namely in the imaging field. The drone presented in this paper will also be used for the delivery of medical supplies. With a lock system implemented on the container attached, it ensures the safety of the supplies till they reach the rightful person. The drone could be used by online pharmaceuticals, which are booming as people are gaining interest in them. The designed system is an internet-based drone using the principles of IoT.
\end{abstract}

\section{Introduction}

Nowadays, the use of drones is increasing in multiple fields such as military, photography, and delivery purposes. Also, in the medical field, it is observed that a drone is been used to deliver medicines, vaccines to distant places where roadway delivery isn't possible. This has helped a lot in the fast and safe delivery of medical supplies. In this pandemic, the use of a drone has increased because of the contact-less delivery, which has hugely impacted society.

Drones used for commercial purposes become more popular when Amazon announced it intends to use drones for delivering packages to their customers. This is a very fascinating and life-changing idea with many intended and unintended consequences [1].

Drones have already been used to deliver small aid packages to places affected by major disasters, including the 2010 earthquake in Haiti, the 2012 hurricane called Superstorm Sandy that affected the northeastern United States, Canada, and the Caribbean, the 2015 category 5 cyclone named Pam that struck the islands of Vanuatu, and the 2015 earthquake (Gorkha) in Nepal. Supplies like medicine, defibrillators, blood samples, and vaccines were being delivered [2], [3], [4].

In rural areas where the mode of roadways delivery isn't possible because of poor road infrastructure and where poor weather the vehicles will not be able to transport the medicines and vaccines. Hence, people might be left without essential medical supplies. So drones can easily navigate through airways and above blocked roads to deliver essential supplies safely and quickly. The drone will be not affected by the road's infrastructure and it will transport the medicines to the people who are living in such ru-

\footnotetext{
*e-mail: pkhandagale02@ gmail.com

**e-mail: mandareranjikal@gmail.com

***e-mail: siddhiparabs00@gmail.com

****e-mail: garg.bhawana@ rait.ac.in
}

ral areas in no time as compared to other vehicles. Drone delivery is a fast, cost-effective strategy to ensure rural patients don't need to go without.

During the medicines transit process, the packages are vulnerable to getting lost or stolen. At times, packages are damaged during the process of delivery. This can cause considerable damage if the package carries some medicines or vaccines in fragile containers. Newly emerging online pharmacies require a faster and secure way of medical transportation. Since, the traditional process is land based, the there is a high risk for medicines getting stolen or smuggled. This may also lead to black marketing of the medicines which are in high demand.

Considering the pandemic scenario caused due to COVID19, the Remdesivir injections and other in-demand antibiotics and anti-body medications required for treatment of patients were falling short in supplies to the patients which were in need. Also, these medicines were sold in unauthorized ways at a much higher rate than the Maximum Retail Price marked on them. For such conditions, a check on supplies and inventory management by a centralized is needed. Also, delivery to the person in need must be ensured.

Patients being treated for diseases like Diabetes, Asthma, cardiac-related diseases, etc. which require a daily dose of medicines and cannot be skipped, at times run out of supplies. In such situations, an urgent delivery of medicines is required[5].

1. Reduces response time during emergency.

2. Serves as an excellent medi-aid.

3. Medkit box attached to transport medicines.

4. Locking mechanism ensures safety of medicines.

5. Prevents medicine contamination.

The next section covers the block diagram of the drone with the locking mechanism used for the container. The fourth section gives technical insights into all the hardware and software used for the drone. In section 5 , the 


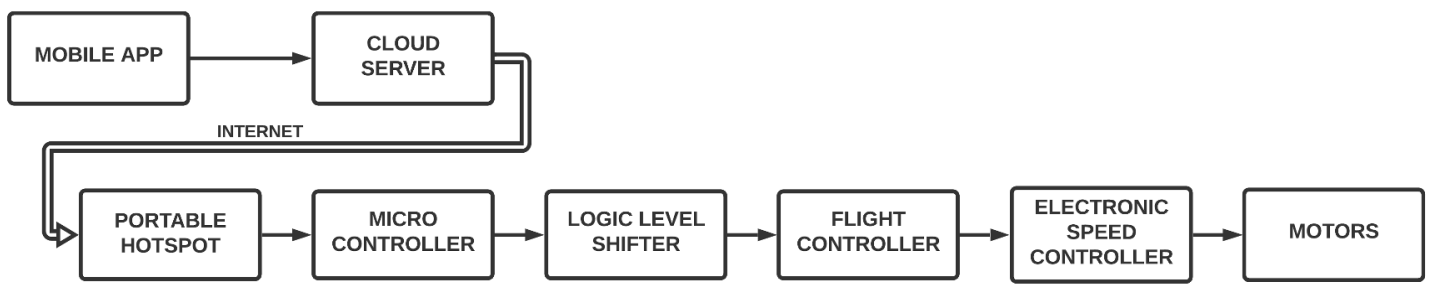

Figure 1. Block Diagram of Drone

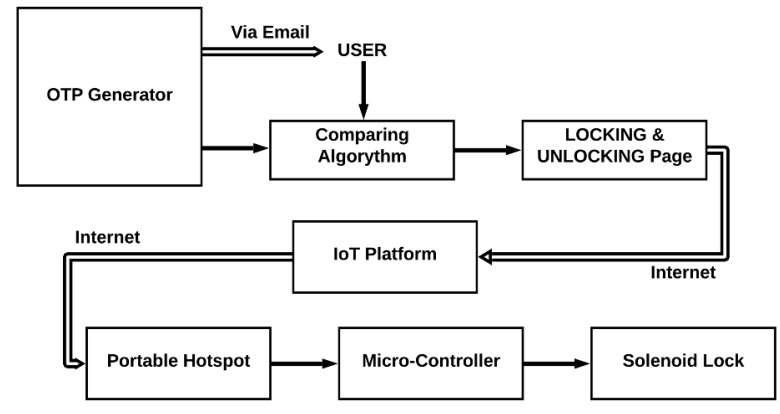

Figure 2. Block Diagram of Locking Mechanism

complete design and the components implementation are reviewed. The sixth section will consist of the snaps taken of the constructed drone. The final section consists of the concluding remarks. The references are also mentioned at the end.

\section{Proposed Work}

The system consists of Flight Controller, ESP8266 microcontroller, Electronic Speed Controller (ESC), and four Brushless DC motors (BLDC) assembled on a quadcopter frame. The system is installed with an onboard portable hotspot device to provide internet connectivity through WiFi. Logic Level Shifters are used for interfacing 3.3V logic with $5 \mathrm{~V}$ logic.

The connectivity between the drone and the remote controller routes via an Internet of Things platform. The remote controller is replaced by a mobile application that holds buttons and a joystick to simulate and produces values similar to a Radio Frequency based remote controller.

The process of controlling the drone starts with a mobile-based remote controller. Primarily, the parameters required for controlling the flight dynamics of the drone are generated via the user interface on the remote controller app. The generated parameter values are uploaded to a specific channel of the server provided by the Internet of Things platform.

The parameter values uploaded to the server are available to be read by the drone. The drone is provided with an onboard portable hotspot. The portable hotspot produces a WiFi zone around the drone which also propagates along with the drone. The portable hotspot enables the drone to connect with the internet to read the parameter values produced and upload by the mobile application.
To read the parameter values, an ESP8266 microcontroller is employed. ESP8266 having an onboard WiFi module that connects with the portable hotspot device to gain access to the internet. Once the ESP8266 is online, it can start reading data from the Internet of Things server. The ESP also serves as the receiver for the drone. On reading the parameter values which were previously uploaded by the mobile application, the ESP microcontroller starts generating PWM (Pulse Width Modulation) signal to represent the parameter values. These generated PWM signals are similar to the PWM signals that would be generated by a traditional receiver. Generation of PWM signals represents the success of the transfer of data from mobile (remote controller) to the drone which is traditionally done by a radio transmitter and receiver [6].

The PWM signals generated by the ESP8266 are fed to respective channels of the Flight Controller of the drone. Hence, the Flight Controller can be controlled by the remote controller on the mobile application.

The Flight Controller controls the flight dynamics of the drone. It drives the Electronic Speed Controllers (ESC). The ESCs acts as an interface between the Flight Controllers and the motors. The ECS regulates the speed of the Brushless DC Motor (BLDC Motor) by the PWM signals fed by the ESP8266. The BLDC motors are fitted with propellers to generate lift. Thus, the drone is controlled by the remote mobile application via the internet [7].

\section{FOR LOCKING MECHANISM:}

The locking mechanism is controlled by a desktop application. The locking vessel consists of an ES8266 microcontroller which operates a solenoid lock or a servo-motor in a combination with a door latch. 


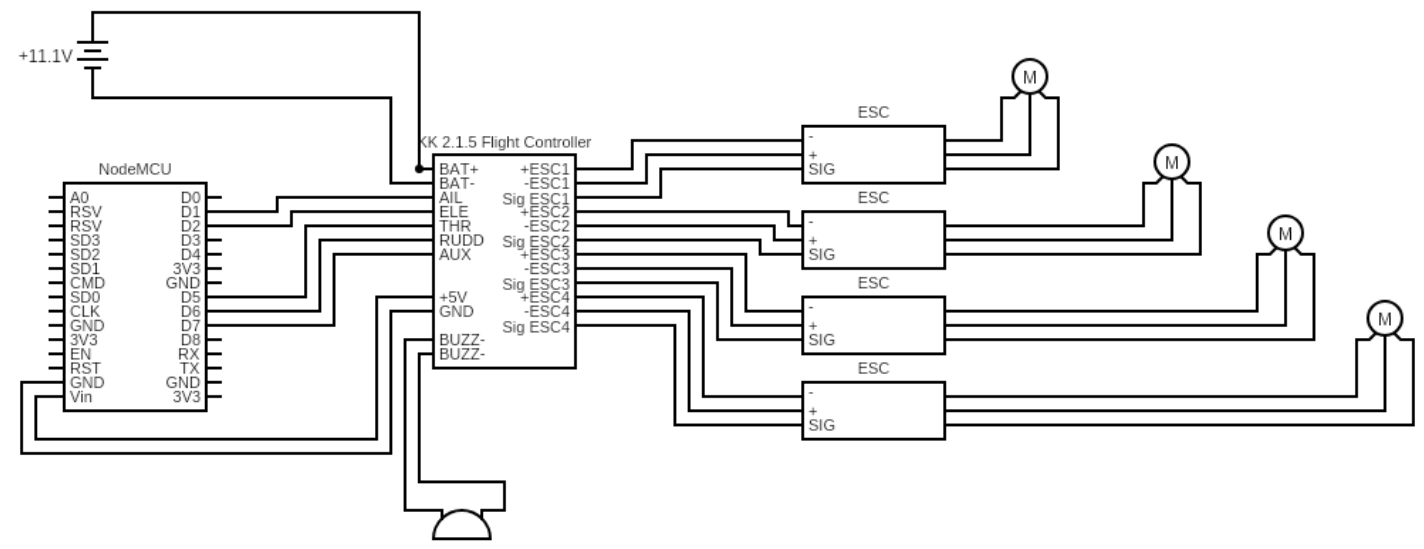

Figure 3. Circuit Diagram for Drone

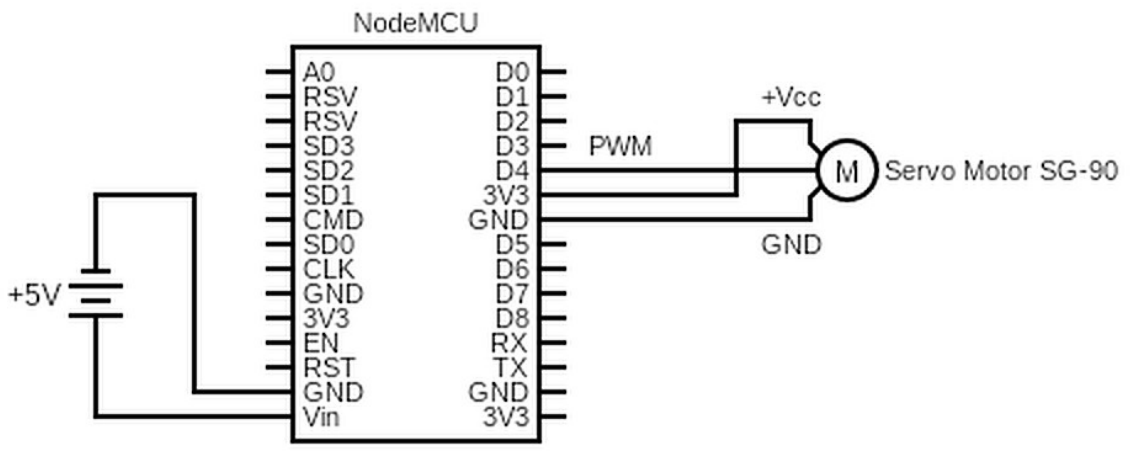

Figure 4. Circuit Diagram for Locking Mechanism

The desktop application generates a One Time Password (OTP) which is sent to the user's email address for verification. It then compares the OTP entered by the user with the OTP which was previously generated by the application. The desktop application establishes a connection with an Internet of Things (IoT) platform. The Lock and Unlock functions send a secret code that represents lock and unlock order. The generated code is sent to the server allotted by the IoT platform. The onboard ESP8266 reads the code uploaded by the application and performs the desired operation on locking or unlocking the container box in accordance with the code sent by the application. The micro-controller controls a solenoid lock.

In this paper, a drone is been proposed that can be used in the medical field.

When there is an urgent need of medicines to a patient who can't go out themselves and bring medicines, or when due to some reason the patient forgets to buy medicines, this drone will deliver the medicines in a box to the patient's location.

Chances of the medicines getting contaminated will be less and the patient will be satisfied [8].

\section{Technical Insights}

\subsection{Hardware Implemented}

Frame: The frame of a quadcopter is the main structure, or the skeleton upon which the rest of components will be attached.

Flight Controller: The Flight Controller (aka "FC") is the brain of a quadcopter, it has sensors on the board so it can understand how the craft is moving.

ESP8266: It is a microcontroller which helps us to connect with the wifi, or send or receive data. With the respective of the project, the ESP is employed as a receiver. It produces PWM signals similar to the signals produced by the receiver output for corresponding values sent by the transmitter.

Electronic Speed Controller: An ESC is a device that interprets signals from the flight controller, and translates those signals into phased electrical pulses to determine the speed of a brushless motor.

Motors: The motors are the main drain of battery power on your drone.

The propellers: The propeller converts the motion into lifting power. Because of the special shape of the blades, the 
air pressure is uneven on two sides while they are in motion, what creates lifting power. The principle can be easily modelled by Newton's third motion law and Bernoulli's principle [9].

Battery: LiPo batteries are the power sources of the quadcopters. LiPo is used because of the high energy density and high discharge rate.

Logic Level Shifter : It is a bi-directional circuit which converts $3.3 \mathrm{~V}$ logic to $5 \mathrm{~V}$ logic or vice-versa.

JioFi Portable Hotspot: It is a device which creats the wifi signal. It is used to connect our project to the internet and the cloud-based server.

Microcontroller: It will be used to manipulate the data and controlling the lock.

Servo-motor SG90: It is used for activating and deactivating lock.

Enclosure: It's an object for holding or transporting something.

\subsection{Software Implemented}

Blynk App: Blynk is a new platform that allows you to quickly build interfaces for controlling and monitoring your hardware projects from your iOS and Android device.

Arduino IDE: The Arduino Integrated Development Environment - or Arduino Software (IDE) - contains a text editor for writing code, a message area, a text console, a toolbar with buttons for common functions and a series of menus. It connects to the Arduino and Genuino hardware to upload programs and communicate with them.

\section{Design and Implementation Details}

\subsection{Design Methodology}

2200 mAh Lipo Battery for the main power supply is used for the flight controller of the drone and KK2.15 Flight controller as the brain of the drone. Also, the controller's $\mathrm{ESC} 1, \mathrm{ESC} 2, \mathrm{ESC} 3$, and ESC4 ports are used to give power supply to the motors. NodeMCU is also employed to operate the drone via $\mathrm{WiFi}$. The $\mathrm{D} 1$ port is connected AIL port, D2 is connected to the Elevator port. Then, D5 was connected to the Throttle. D6 was connected to the rudder and D7 connected to the Auxiliary pin of the Flight Controller. Vin was connected to a $5 \mathrm{~V}$ supply while both the ground pins are also connected. The buzzer is also connected to the Flight Controller for signaling. The motors are also connected to the propellers and the power supply was given by the ESC. The Servo Motor has 3 pins, Vcc, ground, and PWM. We connected Servo Motor's ground pin to NodeMCU's ground pin and PWM pin to the D4 pin. D4 pin is allocated to generate a PWM signal equivalent to the desired angle for locking and unlocking the box. The VCC for the Servo motor is provided through the +3.3 $\mathrm{V}$ on-board supply of NodeMCU. Arms of the Servo motors are connected to the handle of the latch using a metal frame that acts as a lever. The Blynk app serves as an interface to operate the microcontroller which in turn controls the locking mechanism.

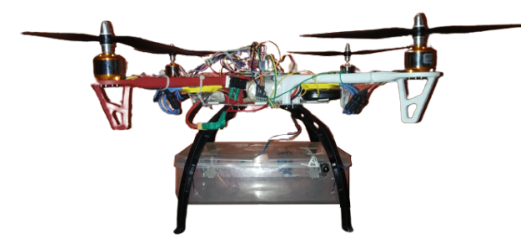

Figure 5. The implemented Drone with container attached (1)

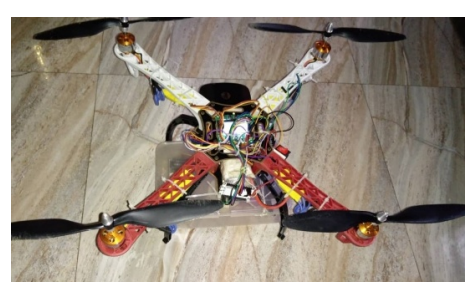

Figure 6. The implemented Drone with container attached (2)

\subsection{Selection Criteria for Components:}

To cut down the cost, an 11.1V 2200mAh Li-po Battery was employed. Also, NodeMCU was used as a transmitter rather than an Arduino because it had Wifi-module and it is less costly than Arduino. A $30 \mathrm{~V}$ ESC battery to power supply the motors were utilized. Also, a Flight Controller KK2.1.5 was used because it has an onboard gyroscope and display for configuration setup. Also, it is inexpensive as compared to other flight controllers in the market. A stand for the drone to lift it from the ground for ground clearance was attached. The plastic box was used because it's lightweight and durable. Also, it can be carried easily by our drone.

\section{Results and Discussion}

As planned, the drone will deliver the required pharmaceutical products to the patient's location.

Just like this, the drone can be used by doctors to transfer medicines to a region where roadways are not good for transport.

While making this drone, major issues arose during connecting the NodeMCU to the flight controller. Also when the drone was taking off, it was quite unstable and because of that, the drone was tilted towards one side. So to overcome the issue, the flight controller was programmed again and reduced the previous values. After these changes, the drone was able to successfully take off. The drone can carry around 1 kilogram including the medkit box containing the medicines. The payload in the Medkit box is considered to be 250 gms for one flight and it will transport the medicines in roughly around 50 minutes. Considering the Medkit box size and structure, it will be able to carry around 750 to 800 grams of medicines in it. As the payload is increased, the time will also increase simultaneously.

Approach time can be reduced and a patient's life will be saved. 
Table 1. Payload associated with Time

\begin{tabular}{ll}
\hline Payload(gm) & Time(mins) \\
\hline 250 & 50 \\
350 & 80 \\
450 & 110 \\
550 & 140 \\
650 & 170 \\
750 & 200 \\
\hline
\end{tabular}

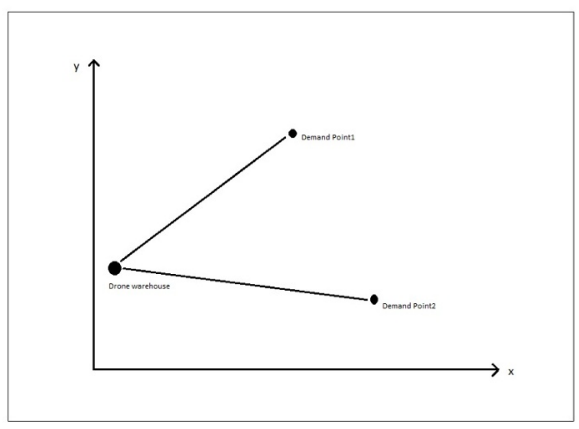

Figure 7. Location of the warehouse and demand zone

In rural areas, a warehouse will be set up to operate, charge and maintain the drone. In case of emergency, one can contact the warehouse near the area and the operator can put on the medical supplies in the Medkit box, and then he can fly the drone to the demand locations. The drone can also cover multiple demand locations in a single flight.

\section{Acknowledgements}

With great pleasure, we avail this opportunity to express our profound gratitude and deep regards to our Head Of Department of Instrumentation Engineering, RAIT, Dr. Sharad P. Jadhav, for his generous support and constant encouragement. It helped us to work in the right direction to attain the desired objective.

\section{Conclusion}

Drones can provide beneficial and humanitarian applications, especially related to healthcare. Furthermore, extreme weather and city congestion can be an obstacle to emergency medical delivery. Consequently, drone healthcare delivery to inaccessible locations is likely to become more present in the near future, in any disaster the medical supplies and basic needs supply is mostly transported using vehicles, by this people will not be able to receive proper help and will have to wait for transportation systems to arrive in their area. So, drones will be a great help in such disastrous situations in supplying medicines and other medical supplies to the people. This paper discusses some innovative applications of drones in healthcare. Healthcare costs are increasing day by day in both developed and developing countries. Since time is of the essence in an emergency, a faster response would prevent medical trauma and potentially save lives. Our models could be used by drone companies, delivery companies, healthcare organizations, humanitarian organizations, or governments. In some cases, governments could partner with drone companies, such as Pharmeasy and Netmeds. Drone healthcare delivery is a new field with many opportunities for impactful future research.

Considering the drone's future scope, a GPS will be added to track the location of the drone and to recover the drone from a lost location in case of any system or power failure. Multiple Medkit boxes could be added in order to deliver medical supplies to multiple demand points in a single flight. High powered batteries could be installed later in order to receive the long flight duration.

\section{References}

[1] Amukele, Timothy, Current state of drones in healthcare: challenges and Opportunities 2, 296-298 (2019)

[2] Balasingam, Manohari, Drones in medicine - the rise of the machines 71, e12989(2017)

[3] Scott, Judy and Scott, Carlton, Drone delivery models for healthcare (2017)

[4] MStalberg, Malachi, Designing and Developing a Commercial Delivery Drone 1, (2020)

[5] Bhatt, Kunj and Pourmand, Ali and Sikka, Neal, Targeted applications of unmanned aerial vehicles (drones) in telemedicine 24, 833-838 (2018)

[6] Mesquita, João and Guimarães, Diana and Pereira, Carlos and Santos, Frederico and Almeida, Luís, Assessing the ESP8266 WiFi module for the Internet of Things 1, 784-791 (2018)

[7] Cheng, Zhuoqun and West, Richard and Einstein, Craig, End-to-end analysis and design of a drone flight controller 37, 2404-2415 (2018)

[8] Zailani, Mohamed Afiq Hidayat and Sabudin, Raja Zahratul Azma Raja and Rahman, Rahana Abdul and Saiboon, Ismail Mohd and Ismail, Aniza and Mahdy, Zaleha Abdullah, Drone for medical products transportation in maternal healthcare: A systematic review and framework for future research, 99, 36(2020)

[9] Dalai, Rama Prasanna and Sabyasachi, Sidharth, Future of Drone Technology in India 1673, 064X

[10] A. Josephin Arockia Dhivya, J. Premkumar, Quadcopter based technology for an emergency healthcare, 1-3 (2017) 